\title{
Discovery and validation of the tumor-suppressive function of long noncoding RNA PANDA in human diffuse large B-cell lymphoma through the inactivation of MAPK/ERK signaling pathway
}

\author{
Yingjun Wang ${ }^{1}$, Mingzhi Zhang ${ }^{1}$, Huanan $X u^{2}$, Yifei Wang ${ }^{3}$, Zhaoming Li $^{1}$, Yu \\ Chang ${ }^{1}$, Xinhuan Wang ${ }^{1}$, Xiaorui Fu ${ }^{1}$, Zhiyuan Zhou ${ }^{1}$, Siyuan Yang ${ }^{1}$, Bei Wang ${ }^{1}$ and \\ Yufeng Shang ${ }^{1}$ \\ ${ }^{1}$ Department of Oncology, The First Affiliated Hospital of Zhengzhou University, Lymphoma Diagnosis and Treatment Center \\ of Henan Province, Zhengzhou, Henan, China \\ ${ }^{2}$ Department of Anorectal Surgery, The First Affiliated Hospital of Zhengzhou University, Zhengzhou, Henan, China \\ ${ }^{3}$ Department of Ultrasonography, The First Affiliated Hospital of Zhengzhou University, Zhengzhou, Henan, China
}

Correspondence to: Mingzhi Zhang, email: Zhangmingzhi_11@163.com

Keywords: IncRNAs, diffuse large B-cell lymphoma, IncRNA PANDA, p53, MAPK/ERK

Received: June 30, $2017 \quad$ Accepted: July 26, $2017 \quad$ Published: August 07, 2017

Copyright: Wang et al. This is an open-access article distributed under the terms of the Creative Commons Attribution License 3.0 (CC BY 3.0), which permits unrestricted use, distribution, and reproduction in any medium, provided the original author and source are credited.

\section{ABSTRACT}

Diffuse large B-cell lymphoma (DLBCL) is one of the leading causes of cancerrelated mortality, and responds badly to existing treatment. Thus, it is of urgent need to identify novel prognostic markers and therapeutic targets of DLBCL. Recent studies have shown that long non-coding RNAs (IncRNAs) play an important role in the development of cancer. By using the next generation HiSeq sequencing assay, we determined IncRNAs exhibiting differential expression between DLBCL patients and healthy controls. Then, RT-qPCR was performed for identification in clinical samples and cell materials, and IncRNA PANDA was verified to be down-regulated in DLBCL patients and have considerable diagnostic potential. In addition, decreased serum PANDA level was correlated to poorer clinical outcome and lower overall survival in DLBCL patients. Subsequently, we determined the experimental role of IncRNA PANDA in DLBCL progression. Luciferase reporter assay and chromatin immunoprecipitation assay suggested that IncRNA PANDA was induced by p53 and p53 interacts with the promoter region of PANDA. Cell functional assay further indicated that PANDA functioned as a tumor suppressor gene through the suppression of cell growth by a G0/G1 cell cycle arrest in DLBCL. More importantly, Cignal Signal Transduction Reporter Array and western blot assay showed that IncRNA PANDA inactivated the MAPK/ERK signaling pathway. In conclusion, our integrated approach demonstrates that PANDA in DLBCL confers a tumor suppressive function through inhibiting cell proliferation and silencing MAPK/ERK signaling pathway. Thus, PANDA may be a promising therapeutic target for patients with DLBCL.

\section{INTRODUCTION}

Diffuse large B-cell lymphoma (DLBCL) occurs most commonly in all subtypes of non-Hodgkin lymphoma (NHL), representing more than one-third of all diagnosed NHL cases and making it the most prevalent form of NHL among adults worldwide $[1,2]$. The administering of the regimen including rituximab plus cyclophosphamide/ doxorubicin/vincristine/prednisone (R-CHOP) has been seemed as the standard therapy for the patients with DLBCL, remarkably improves the prognosis [3, 4]. However, a great amounts of patients still suffer from unsatisfactory prognosis [5]. Thus, it is important to investigate novel biomarkers involved in DLBCL development.

Gene expression profiling of specimens from DLBCL has revealed broad gene expression deregulation 
compared to healthy individuals. It is more and more clear that most of the genome DNA is represented in processed transcripts without or lacking of proteincoding capacity [6]. Long noncoding RNAs (lncRNAs) have been implicated in a variety of physiological and pathological processes. In cancer, aberrant expression and mutations of IncRNAs can contribute to tumor development and progression by promoting proliferation, invasion, metastasis, and survival [7, 8]. Several groups have performed systematic analysis of lncRNAs by Hiseq sequencing array, a high throughput screening method, in normal cells and also in primary tumors. A recent cross-cancer study by the Chinnaiyan group uncovered thousands of novel lncRNAs [9]. Another study by the Maher group identified a large number of novel lncRNAs in lung cancer [10].

Dysregulation of some lncRNAs is well accepted as prognostic factor and therapeutic target for specific cancers. For example, HOTAIR contributes to the tumorigenesis of breast cancer [11], MALAT1 is attributed to the metastasis of non-small cell lung cancer [12], and HULC is identified as critical regulator of pancreatic cancer [13]. The long non-coding RNA PANDA (P21 Associated ncRNA DNA Damage Activated) is located $\sim 5$ $\mathrm{kb}$ upstream of the CDKN1A (p21) transcription start site, is evolutionarily conserved, specifically induced by DNA damage and mediates proliferation/apoptosis functions [14]. It is reported that PANDA positively regulates proliferation of osteosarcoma cells [15]. However, the patterns and biological function of IncRNA PADNA in DLBCL is still unclear.

In this study, a high-throughput Hiseq sequencing was firstly performed to find the potential aberrant lncRNAs. Then, the reverse transcription quantitative real-time PCR (RT-qPCR) assays were used to validate the upregulation or downregulation of those lncRNAs in both clinical samples and cell materials. We reveals that lncRNA PANDA is downregulated in DLBCL patients and the suppression of PANDA indicates poor outcome. Moreover, lncRNA PANDA can inhibits proliferation of DLBCL cells through the inactivation of MAPK/ERK signaling pathway.

\section{RESULTS}

\section{Discovery of potential IncRNAs by Hiseq sequencing method}

We firstly screened potential lncRNAs that differentially expressed between DLBCL patients and healthy individuals via Hiseq array in the discovery phase. On the basis of the date obtained from Hiseq sequencing, we then identified 546 lncRNAs that were differently expressed more than 2-fold change. Subsequently, we narrowed the scope of the study to the 120 most aberrant expressed RNAs, including 60 up-regulated lncRNAs and
60 down-regulated lncRNAs (Figure 1). These lncRNAs should be easily detected with a $\mathrm{Ct}$ value less than 35 , easily designed primers and have stable experssion in both primary tissues and serum samples. According those definition, we finally restricted to $10 \operatorname{lncRNAs}$ from 5 of up-regulated group and 5 of down-regulated group (Table 1). In addition, another five lncRNAs were brought into our study, as they were reported to have potential function during DLBCL progression [16-20]. Thus, we discovered 15 candidate lncRNAs which may be potential biomarkers for DLBCL patients, pending further validation.

\section{LncRNA PANDA was downregulated in DLBCL patients}

RT-qPCR was performed to further test the 15 lncRNAs selected through the Hiseq sequencing method by using 40 primary DLBCL tissues and 40 noncancerous lymph node tissues. Among these, six lncRNAs (TUG1, HOTAIR, HULC, PANDA, FLJ46300, LincRNA-p21) were found significantly dysregulated (Table 2). Subsequently, these six genes were further determined by RT-qPCR in the validation phase containing 68 serum samples from patients with DLBCL and 68 from healthy controls. In this phase, we observed that one lncRNA PANDA was significantly down-regulated in DLBCL patients compared with healthy controls, while another lncRNA TUG1 expression was dramatically increased in DLBCL patients (Figure 2A). Then, we detected the expression of IncRNA PANDA and TUG1 in DLBCL cell lines. We found that PANDA was significantly down-regulated in the five DLBCL cell lines compared with normal cells, while no statistical significance was found for the expression of TUG1 in different cell lines (Figure 2B). More importantly, previous studies showed that PANDA may participated in tumorigenesis of several malignancies through the regulation of cell proliferation [15]. Thus, we focus on the clinical and experimental role of lncRNA PANDA in DLBCL.

\section{Decreased expression of serum IncRNA PANDA indicates poor outcome of DLBCL patients}

After having validated the down-regulation of lncRNA PANDA, we investigated the association between serum PANDA expression and clinicopathological characters. As shown in Table 3, lncRNA PANDA was significantly correlated with B symptoms, Ann arbor stages, CHOP-like treatment, Rituximab and IPI, while no significant correlations were observed between PANDA expression and other clinicopathological factors, such as gender, age, performance status and subtypes in 68 serum samples. In addition, a similar conclusion was also developed in the 40 primary tissue samples (Table 4). To evaluate the role of PANDA in distinguishing between the 
Table 1: Candidate IncRNAs selected on a basis of the Hiseq analysis

\begin{tabular}{lcccc}
\hline \multicolumn{1}{c}{ Seq-name } & Location & Regulation (D. vs C.) & Fold change & $\boldsymbol{P}$ value \\
\hline TUG1 & Chr22q12.2 & $\mathrm{Up}$ & 112.1462 & 0.00007984 \\
FIRRE & ChrXq26.2 & $\mathrm{Up}$ & 77.3592 & 0.00016824 \\
SBDSP1 & Chr7q11.23 & $\mathrm{Up}$ & 39.0431 & 0.00082395 \\
GGT8P & Chr2p11.2 & $\mathrm{Up}$ & 31.0532 & 0.00090357 \\
MIR210HG & Chr11p15.5 & $\mathrm{Up}$ & 25.3981 & 0.00163746 \\
FLJ46300 & Chr10q26.3 & Down & 65.4768 & 0.00015396 \\
PANDA & Chr6p21.1 & Down & 43.6921 & 0.00044281 \\
FTX & ChrXq13.2 & Down & 29.4650 & 0.00103498 \\
LOC151009 & Chr2q13 & Down & 17.3986 & 0.00204437 \\
LOC202181 & Chr5q35.3 & Down & 14.6935 & 0.00411932 \\
\hline D.: DLBCL;
\end{tabular}

D.: DLBCL; C.:Controls.

Table 2: Expression of 15 candidate IncRNAs in tissue specimens from DLBCL patients and control individuals [median (interquartile range)]

\begin{tabular}{lccc}
\hline \multicolumn{1}{c}{ LncRNA } & DLBCL & Controls & $\boldsymbol{P}$ value \\
\hline TUG1 & $2.41(1.30-3.44)$ & $1.21(0.40-2.85)$ & $<0.01$ \\
FIRRE & $1.35(0.49-3.08)$ & $0.98(0.30-2.77)$ & 0.10 \\
SBDSP1 & $1.64(0.56-3.28)$ & $1.09(0.36-2.44)$ & 0.25 \\
GGT8P & $1.61(0.72-3.71)$ & $1.32(0.30-2.05)$ & 0.34 \\
MIR210HG & $1.29(0.93-2.57)$ & $1.01(0.41-2.05)$ & 0.12 \\
FLJ46300 & $0.77(0.45-1.93)$ & $1.36(0.42-2.06)$ & $<0.01$ \\
PANDA & $1.02(0.39-2.81)$ & $2.56(0.53-3.88)$ & $<0.01$ \\
FTX & $1.32(0.28-2.33)$ & $1.77(0.65-3.01)$ & 0.09 \\
LOC151009 & $0.98(0.41-1.99)$ & $1.23(0.89-2.42)$ & 0.17 \\
LOC202181 & $0.88(0.30-1.51)$ & $1.30(0.41-2.55)$ & 0.10 \\
HOTAIR & $1.63(0.43-2.37)$ & $0.83(0.27-1.74)$ & $<0.05$ \\
HULC & $1.62(0.45-2.18)$ & $0.94(0.33-1.43)$ & $<0.05$ \\
LUNAR1 & $0.84(0.34-2.71)$ & $0.64(0.23-1.68)$ & 0.29 \\
LINCRNA p21 & $0.82(0.22-1.69)$ & $1.56(0.45-2.83)$ & $<0.01$ \\
PEG10 & $1.24(0.55-1.61)$ & $1.02(0.31-1.78)$ & 0.47 \\
\hline
\end{tabular}

DLBCL patients and healthy controls, ROC curve analysis was performed and the cut-off value was established (1.02). The area under the curve (AUC) and diagnostic sensitivity and specificity reached 0.760, 60.29\%, and $77.94 \%$, respectively (Figure 3A). Then, KaplanMeier survival analysis was performed to investigate the prognostic value of PANDA in DLBCL patients. Our results indicated that patients with high PANDA expression were associated with longer OS and RFS compared with low PANDA patients (Figure 3B and 3C). Furthermore, we performed Cox regression univariate/ multivariate analysis to identify whether PANDA or any other clinical parameters were independent indicators for the overall survival of DLBCL patients. The results indicated that PANDA expression level and Ann Arbor stages status were identified as potential independent prognostic factors for overall survival of DLBCL patients (Table 5).

\section{LncRNA PANDA is activated by transcription factor p53 and p53 interacts with the promoter region of PANDA}

In order to determine why lncRNA PANDA is silenced in DLBCL tissues, we focused on transcription factors that potentially bind to the PANDA promoter. Since p53 is a positive regulator of CDKN1A during the DNA damage response, we asked whether p53 regulates PANDA expression. Based on the computer algorithms PROMO (http://alggen.lsi.upc.es/cgi-bin/ 


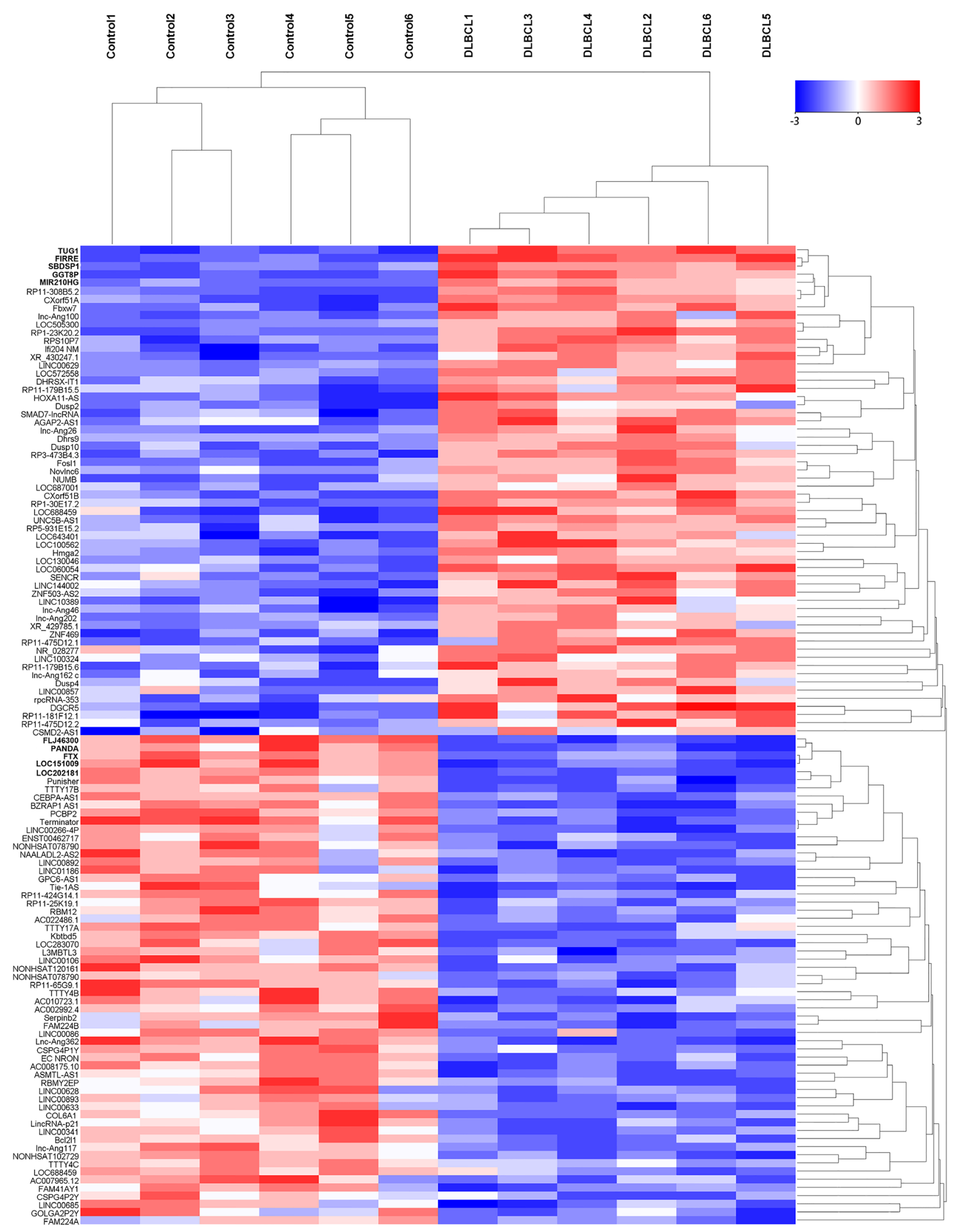

Figure 1: The heat map shows expression of the 120 IncRNAs most up- or down-regulated in DLBCL patients compared with healthy controls. The top 60 lncRNAs up- and down-regulated in non-responding group are shown in the top and bottom halves, respectively. The heat map was generated with an R package using normalization across rows (serum samples). 
Table 3: Association of IncRNA PANDA expression with clinical parameters in DLBCL patients

\begin{tabular}{|c|c|c|c|c|}
\hline & $\begin{array}{c}\text { total } \\
n(\%)\end{array}$ & $\begin{array}{c}\text { High PANDA expression } \\
n(\%)\end{array}$ & $\begin{array}{c}\text { Low PANDA expression } \\
n(\%)\end{array}$ & $P$ value \\
\hline Gender & & & & 0.590 \\
\hline Male & 49 & $26(38.2)$ & $23(33.8)$ & \\
\hline Female & 19 & $8(11.8)$ & $11(16.2)$ & \\
\hline Age, year & & & & 0.825 \\
\hline Median & & 63 & 59 & \\
\hline Range & & $34-85$ & $36-79$ & \\
\hline B symptoms & & & & $0.024^{*}$ \\
\hline Present & 26 & $8(11.8)$ & $18(26.5)$ & \\
\hline Absent & 42 & $26(38.2)$ & $16(23.5)$ & \\
\hline Ann Arbor stages & & & & $0.014^{*}$ \\
\hline I-II & 31 & $21(30.9)$ & $10(14.7)$ & \\
\hline III-IV & 37 & $13(19.1)$ & $24(35.3)$ & \\
\hline Performance status & & & & 0.803 \\
\hline $0-2$ & 26 & $14(20.6)$ & $12(17.6)$ & \\
\hline $3-4$ & 42 & $20(29.4)$ & $22(32.4)$ & \\
\hline CHOP-like treatment & & & & $0.003^{*}$ \\
\hline Response & 33 & $23(33.8)$ & $10(14.7)$ & \\
\hline Non-response & 35 & $11(16.2)$ & $24(35.3)$ & \\
\hline Rituximab & & & & $0.006^{*}$ \\
\hline Response & 38 & $25(36.8)$ & $13(47.1)$ & \\
\hline Non-response & 30 & $9(13.2)$ & $21(19.1)$ & \\
\hline Subtypes & & & & 0.642 \\
\hline $\mathrm{GC}$ & 39 & $18(26.5)$ & $21(30.9)$ & \\
\hline Non-GC & 29 & $16(23.5)$ & $13(19.1)$ & \\
\hline IPI & & & & $0.021^{*}$ \\
\hline $0-2$ & 24 & $7(10.3)$ & $17(25.0)$ & \\
\hline $3-5$ & 44 & $27(39.7)$ & $17(25.0)$ & \\
\hline
\end{tabular}

*, statistical significance.

promo_v3/promo/promoinit.cgi?dirDB $\left.=\mathrm{TF} \_8.3\right), \quad$ and GeneCards (http://www.genecards.org/cgi-bin/carddisp. $\mathrm{pl}$ ? gene $=\mathrm{MYC} \&$ keywords $=$ cmyc), we also identified the p53 binding site on the promoter region of PANDA gene (Figure 4A). We then determined the expression of p53 and found that serum p53 mRNA was down-regulated in DLBCL patients compared with healthy individuals (Figure 4B). p53 protein was also down-regulated in DLBCL cell lines compared with normal B-cell lines (Figure 4C). Moreover, RT-qPCR showed a significant positive correlation between $\mathrm{p} 53$ and PANDA expression in serum samples of DLBCL patients (Figure 4D). LncRNA PANDA expression was significantly increased after p53 was overexpressed in U2932 and OCI-Ly8 cells (Figure 4E and 4F).
To investigate the direct binding of p53 to the PANDA promoter, we cloned the promoter region $(\sim 1.5$ $\mathrm{kb}$ ) of PANDA into luciferase reporter plasmid (pGL4 basic, Figure 4G). As shown in Figure 4H, luciferase activity was significantly increased in wild p53transfected cells compared with control vector in OCILy8 cells, however, no change of luciferase activity we found when the cells were transfected mutant-p53 RNA. ChIP experiments showed that a significant increased immunoprecipitation of $\mathrm{p} 53$ was identifed at the promoter region of PANDA compared with blank IgG controls (Figure 4I). To conclude, we validated that lncRNA PANDA is activated by transcription factor $\mathrm{p} 53$ and $\mathrm{p} 53$ can interact with the promoter region of PANDA gene. 


\section{LncRNA PANDA suppresses proliferation and induces cell-cycle arrest in DLBCL cells}

As a follow-up to our patient data that revealed a lower expression of PANDA in DLBCL, we further investigated the biological function of PANDA in vitro. LncRNA PANDA was silenced or overexpressed by transfection of si-PANDA or PANDA vector, respectively (Figure 5A and 5B). CCK8 assay showed that si-PANDA significantly promoted cell viability while PANDA overexpression suppressed cell proliferation rate of DLBCL cells (Figure 5C and 5D). We also detected the expression of proliferation marker Ki-67 and found that lncRNA PANDA suppressed Ki-67 expression in OCILy8 cells (Figure 5E). Concomitant with this inhibition of cell proliferation by PANDA, the cell cycle analysis indicated that cell cycle arrest reached significance at the G0/G1 checkpoint after over expression of PANDA (Figure 5F). Moreover, PANDA also suppressed the colony formation capacity of DLBCL cells (Figure 5G). However, no significant effect of PANDA on DLBCL cell apoptosis, migration and invasion was found (Date not shown). This suggests that lncRNA PANDA may act as tumor suppressor gene through regulating cell growth with a G0/G1 cell cycle arrest in DLBCL.

\section{LncRNA PANDA regulates cell proliferation through inactivation of MAPK/ERK signaling pathway}

Subsequently, we investigated the underlying regulatory mechanism by which PANDA regulates
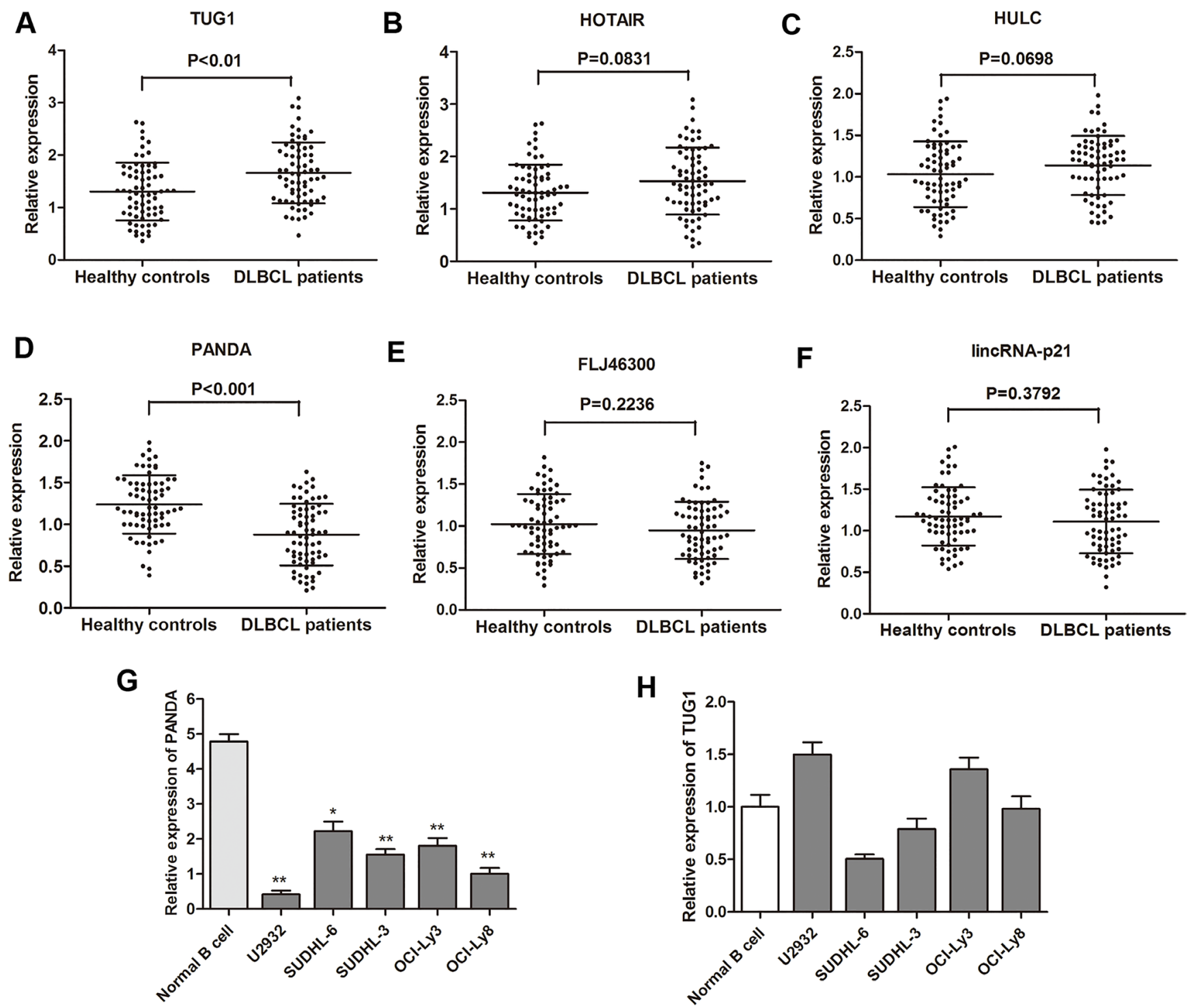

Figure 2: Analysis and validation of selected IncRNA expression by RT-qPCR. (A-F) Concentrations of six identified serum lncRNAs in DLBCL patients $(n=68)$ and healthy controls $(n=68)$ using RT-qPCR assay in validation cohort. $(\mathbf{G}-\mathbf{H})$ The expression levels of lncRNA PANDA and TUG1 were further validated in five DLBCL cell lines and one normal cell line. 
Table 4: Association of IncRNA PANDA expression with clinical parameters in DLBCL tissues

\begin{tabular}{|c|c|c|c|c|c|}
\hline & & $\begin{array}{c}\text { total } \\
n(\%)\end{array}$ & $\begin{array}{c}\text { High PANDA expression } \\
n(\%)\end{array}$ & $\begin{array}{c}\text { Low PANDA } \\
\text { expression } \\
n(\%)\end{array}$ & $P$ value \\
\hline \multirow[t]{3}{*}{ Gender } & & & & & 0.741 \\
\hline & Male & 26 & $12(30.0)$ & $14(35.0)$ & \\
\hline & Female & 14 & $8(20.0)$ & $6(15.0)$ & \\
\hline \multirow[t]{3}{*}{ Age, year } & & & & & 0.749 \\
\hline & Median & & 60 & 61 & \\
\hline & Range & & $37-82$ & $41-79$ & \\
\hline \multirow[t]{3}{*}{ B symptoms } & & & & & $0.022^{*}$ \\
\hline & Present & 16 & $4(10.0)$ & $12(30.0)$ & \\
\hline & Absent & 24 & $16(40.0)$ & $8(20.0)$ & \\
\hline \multicolumn{2}{|c|}{ Ann Arbor stages } & & & & $0.019^{*}$ \\
\hline & I-II & 14 & $11(27.5)$ & $3(7.5)$ & \\
\hline & III-IV & 26 & $9(22.5)$ & $17(42.5)$ & \\
\hline \multicolumn{2}{|c|}{ Performance status } & & & & 0.523 \\
\hline & $0-2$ & 17 & $10(25.0)$ & $7(17.5)$ & \\
\hline & $3-4$ & 23 & $10(25.0)$ & $13(32.5)$ & \\
\hline \multicolumn{2}{|c|}{ CHOP-like treatment } & & & & $0.001^{*}$ \\
\hline & Response & 21 & $16(40.0)$ & $5(12.5)$ & \\
\hline & Non-response & 19 & $4(10.0)$ & $15(37.5)$ & \\
\hline \multirow[t]{3}{*}{ Rituximab } & & & & & $0.001^{*}$ \\
\hline & Response & 23 & $17(42.5)$ & $6(15.0)$ & \\
\hline & Non-response & 17 & $3(7.5)$ & $14(35.0)$ & \\
\hline \multirow[t]{3}{*}{ Subtypes } & & & & & 0.515 \\
\hline & $\mathrm{GC}$ & 25 & $11(27.5)$ & $14(35.0)$ & \\
\hline & Non-GC & 15 & $9(22.5)$ & $6(15.0)$ & \\
\hline \multirow[t]{3}{*}{ IPI } & & & & & $0.022^{*}$ \\
\hline & $0-2$ & 14 & $3(7.5)$ & $11(27.5)$ & \\
\hline & $3-5$ & 26 & $16(40.0)$ & $10(25.0)$ & \\
\hline
\end{tabular}

*, Statistical significance.

DLBCL progression. The Cignal Signal Transduction Reporter Array was performed to determine the change of signaling activity PANDA was overexpressed in OCILy8 cells. Among the 50 candidate signaling pathways, we found that the activity of MAPK/ERK signaling pathway was mostly silenced by PANDA overexpression (Figure 6A). MAPK/ERK signaling pathway participates in the regulation of proliferation and cell cycle in tumors, and it has been well accepted that there are functional interactions between p53 and MAPK/ERK signaling pathway [21]. Herein, we sought to determine if MAPK/ ERK pathway is responsible for the lncRNA PANDAinduced suppression of cell proliferation. As expected, western blot experiments showed that lncRNA PANDA

inhibited the expression of proteins involved in MAPK/ ERK pathway (Figure 6B). In addition, DLBCL cells were treated with MAPK/ERK agonist Anisomycin, and the CCK8 assay showed that treatment with Anisomycin potently abolished the PANDA-induced suppression of cell growth (Figure 6C). Cell proliferation marker Ki67 was also reversed by Anisomycin, suggesting that IncRNA PANDA may regulate DLBCL cell proliferation via MAPK/ERK pathway (Figure 6D).

\section{DISCUSSION}

LncRNAs has emerged as an important regulator with impact on a wide range of cancers [22]. They have 
been identified as novel biomarkers and therapeutic targets for various cancers including DLBCL. In current study, high-throughput HiSeq sequencing was firstly employed to search for potential lncRNAs that may help improve the efficacy of diagnosis and prognosis in DLBCL patients. Then, the selected lncRNAs were detected and validated in different specimens such as primary tissues and serums from DLBCL patients, which can assure a relative high accuracy of the date. LncRNA PANDA was then screened out as the most promising lncRNA among the candidates, and its high diagnostic value was also observed in view of high AUC, diagnostic sensitivity and specificity by ROC curves. Additionally, Kaplan-Meier analysis suggested that lower PANDA expression was correlate with poorer survival rate for patients with DLBCL. Moreover, we demonstrated that IncRNA PANDA was induced by p53 and PANDA can suppress cell proliferation through inactivation of MAPK/ERK signaling pathway.

The aberrant expressions of specific lncRNAs in cancer can mark the spectrum of disease progression and may serve as independent biomarkers for diagnosis and prognosis [23]. Previously, Zhou et al. identified a group of lncRNAs that that may have potential value in diagnosting and predicting DLBCL [24]. However, whether some other lncRNAs can also serve as mature biomarkers in DLBCL is still not well known. For this reason, it is of
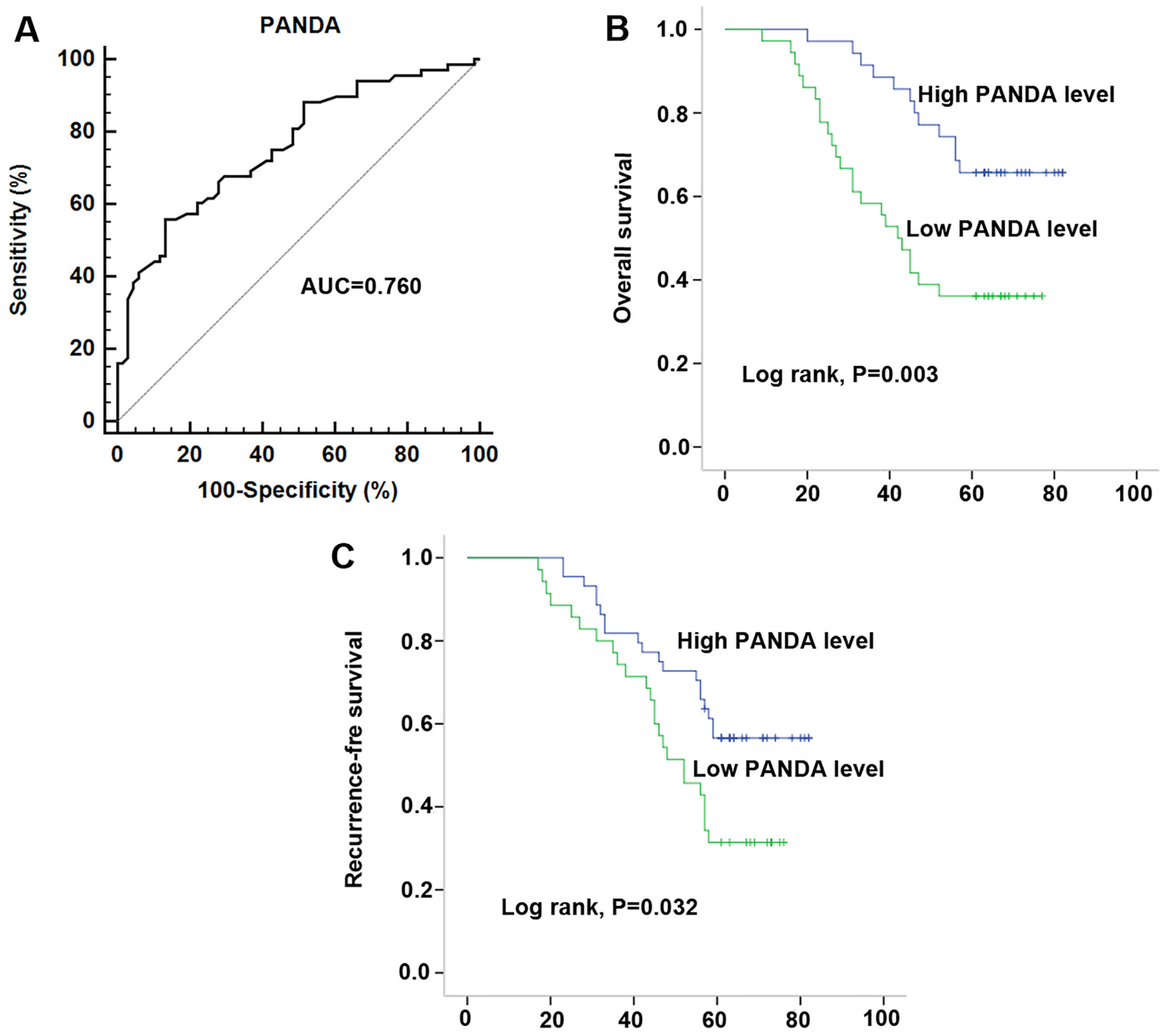

Figure 3: Decreased serum PANDA expression was associated with poor clinical outcome in DLBCL patients. (A) ROC curves for differentiating the DLBCL patients from healthy controls using lncRNA PANDA expression in validation cohort. (B-C) KaplanMeier curves for overall survival (B) and recurrence-free survival (C) according to serum levels of PANDA in DLBCL patients in validation cohort. 
Table 5: Univariate and multivariate Cox proportional hazards regression model analysis of factors for OS in patients with DLBCL

\begin{tabular}{lcccccc}
\hline \multirow{2}{*}{ Characteristics } & \multicolumn{3}{c}{ Univariate analysis } & \multicolumn{2}{c}{ Multivariate analysis } \\
\cline { 2 - 7 } & HR & $\mathbf{9 5 \%}$ CI & $\boldsymbol{P}$ value & HR & 95\%CI & P value \\
\hline Gender & 1.012 & $0.605-2.035$ & 0.682 & & & \\
Age & 1.511 & $0.706-2.902$ & 0.157 & & & \\
Performance status & 1.698 & $0.521-2.906$ & 0.228 & & & \\
Subtypes & 1.883 & $1.127-3.359$ & 0.069 & & & \\
IPI & 1.577 & $0.941-2.694$ & 0.139 & & & \\
Ann Arbor stages & 2.780 & $1.569-3.903$ & 0.009 & 2.791 & $1.475-4.462$ & $0.007^{*}$ \\
B symptoms & 2.173 & $1.133-3.936$ & 0.038 & 2.202 & $1.132-3.308$ & 0.058 \\
PANDA expression & 1.973 & $0.368-2.315$ & 0.006 & 1.893 & $0.384-2.447$ & $0.010^{*}$ \\
\hline
\end{tabular}

*, Statistical significance.
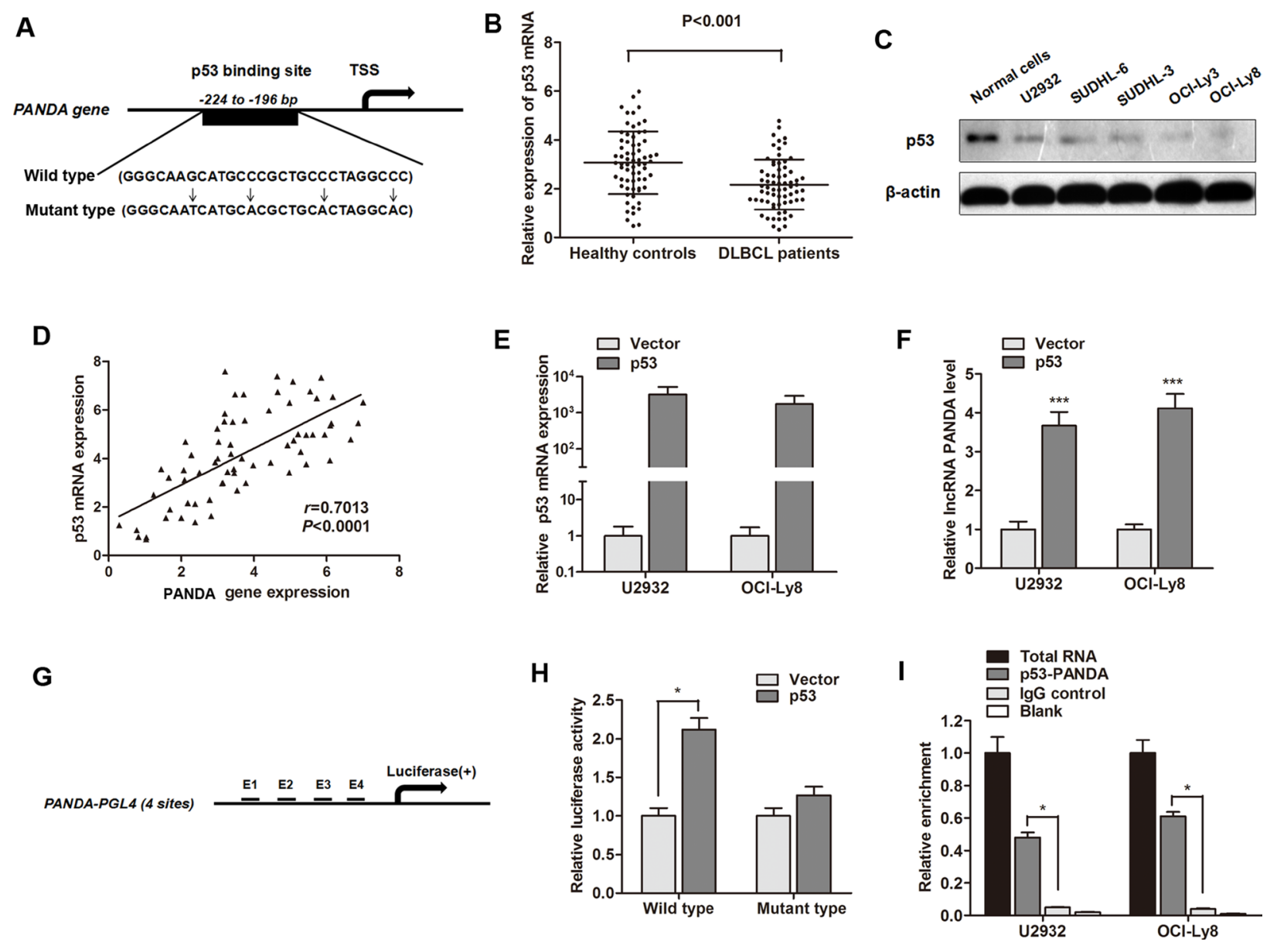

Figure 4: LncRNA PANDA is induced by p53 and p53 interacts with the p53 response element in the promoter region of PANDA. (A) The potential binding site of p53 at the promoter region of IncRNA PANDA, and the description of wild p53 and mutant p53. (B) RT-qPCR showed that p53 mRNA was significantly decreased in serum of DLBCL patients compared with healthy individuals. (C) Western blot experiment indicated that p53 protein was dramatically silenced in most DLBCL cell lines compared with normal cells. (D) Spearman correlation assay suggested a positive correlation between p53 mRNA and MALAT1expression. (E) p53 was significantly elevated by the transfection of p53 overexpression vector. (F) lncRNA PANDA was significantly up-regulated by p53. (G) Potential p53 binding region in the promoter region of PANDA used for construction of luciferase vector containing the binding region. (H) Luciferase activity was significantly increased in p53-transfected cells compared with control vector in OCI-Ly8 cells. (I) The p53 binding at the promoter regions of PANDA was assessed by ChIP analysis. Shown are representative images of three independent experiments. 
utmost importance to identify molecular bio-markers that have effective diagnostic and prognostic meaning. In this study, we sought to identify one or more lncRNAs that may have function in the formation of DLCBL and its progression by unabridged research strategies. As we know, one lncRNA may expressed different levels in different samples and different diseases. Thus, different clinical materials were used to ensure that the selected lncRNAs are qualified to the use in clinical practice. Moreover, we enrolled a relatively large sample group to ensure the steady expression of candidate lncRNAs. With this unabridged research strategy, we identified only one significantly altered lncRNA, PANDA.

LncRNA PANDA was firstly identified by Hung et al. and located approximately 5 kilobases upstream of the p21 TSS, coincides with a cluster of previously annotated ESTs, and is evolutionarily conserved and potentially regulated by p53 [22]. Specifically, PANDA is a 5'-capped and polyadenylated non-spliced lncRNA that is transcribed antisense to $\mathrm{p} 21$ but not dependent on $\mathrm{p} 21$. It
A

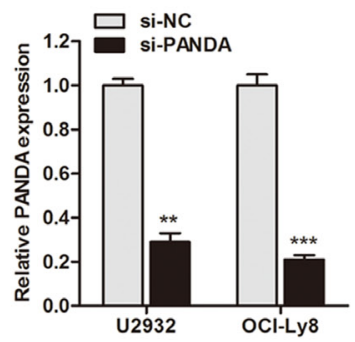

D

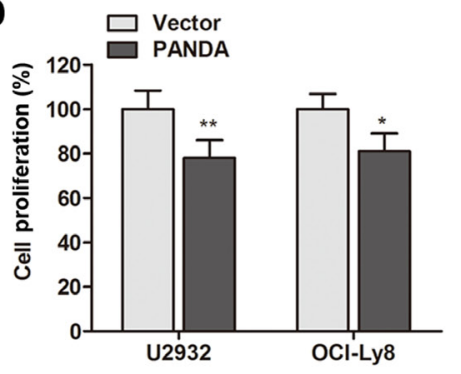

B

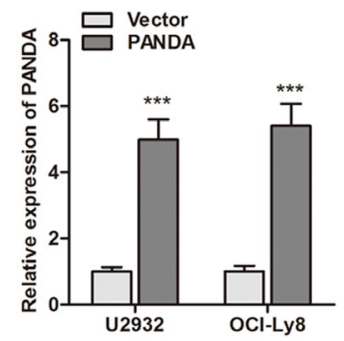

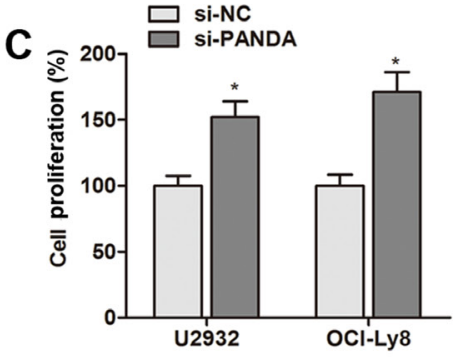

$\mathbf{E}$
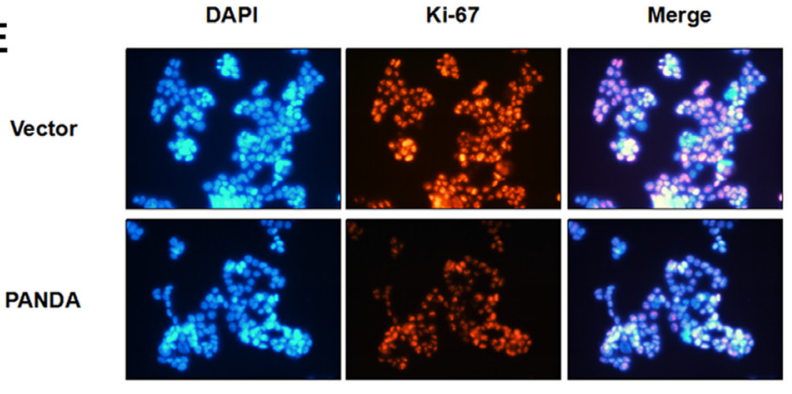

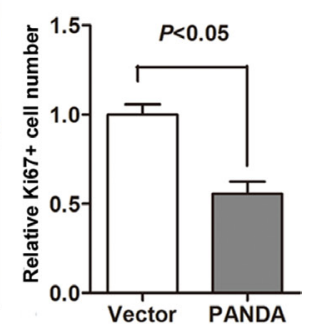

$\mathbf{F}$
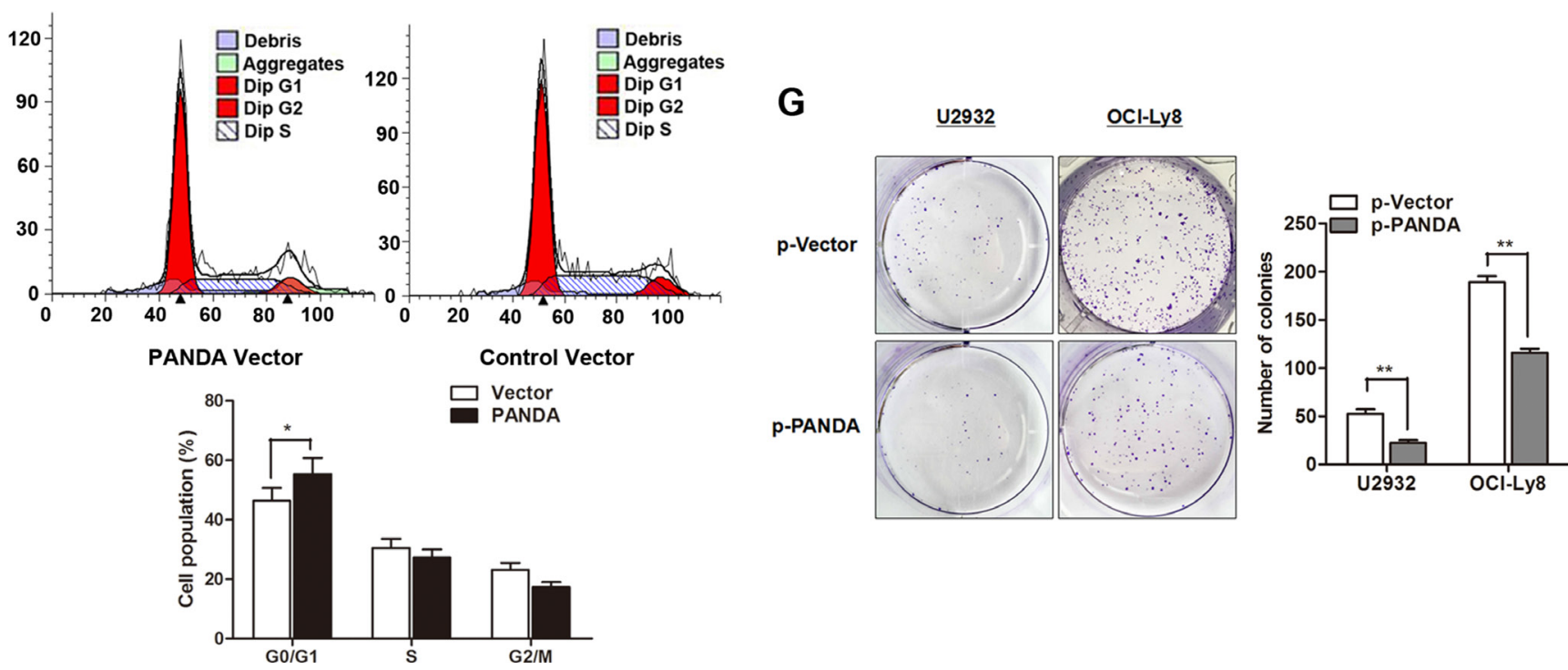

Figure 5: LncRNA PANDA suppresses proliferation and induces cell-cycle arrest in DLBCL cells. (A-B) PANDA expression was significantly silenced by PANDA inhibitor (A) or promoted by PANDA vector (B). (C-D) si-PANDA significantly promoted cell proliferation rate (C) while PANDA overexpression suppressed cell growth (D). (E) Immunofluorescence analysis PANDA overexpression dramatically suppressed the cell proliferation marker Ki-67 protein. (F) Cell-cycle analysis indicated that overexpression of PANDA promoted cell cycle arrest in G0/G1 phase in OCI-Ly8 cells. (G) LncRNA PANDA suppressed the colony formation capacity of U2932 and OCI-Ly8 cells. 
is reported to repress apoptosis by inhibiting the function of nuclear transcription factor Y subunit alpha (NF-YA). In addtion, it can interact with $\mathrm{p} 53$ and stabilize $\mathrm{p} 53$ protein in response to DNA damage. p53 is a transcription factor and then induced by cellular stress regulates proliferation, cell cycle arrest and apoptosis [25]. Published data also demonstrated that $\mathrm{p} 53$ participates in the regulation of neuronal differentiation. Our date suggests that PANDA is induced by translational factor p53 in DLBCL, and p53 can specifically activate PANDA expression through binding to PANDA promoter region.

Previous studies about the function of PANDA in cancer progression are very limited. Puvvula PK et al. indicated that PANDA can positively regulate cell senescence entry and exit via scaffold-attachment-factor SAFA, however, this does not develop a consistency [26]. To the best of our knowledge, we have reported for the first time that lncRNA PANDA can suppress proliferation of DLBCL cells with a G0/G1 cell cycle arrest manner, but had little effect on apoptosis and metastasis. To reveal how lncRNA PANDA participates in the regulation of cell proliferation, we performed Cignal Signal Transduction Reporter Array. This array involved a mixture of a pathway-specific transcription factor-responsive firefly luciferase reporter, which contains a specific transcription factor-responsive element in the promoter. This highthroughput dual-luciferase assay leads us to identify
MAPK/ERK pathway as one putatively affected by lncRNA PANDA. There are three major subfamilies of MAPKs, including p38, ERKs, JNKs, which positively participated biological processes such as migration, proliferation and angiogenesis. The MAPK/ERK signaling pathway is a main signal transduction pathway closely connected with several stress reactions, and physical and chemical reactions within the cells. It was involved in the cell response to outside stimuli by activating and regulating the client protein [27]. Moreover, a wide range of studies have found that the MAPK/ERK pathway closely interacted with p53 pathway [28], which is consistent with the date obtained from the high-throughput dual-luciferase assay.

Aberrant activation of the MAPK/ERK signaling pathway has been observed in many types of human cancers including DLBCL [29]. It mediates cancer cell invasion and proliferation by orchestrating several key biological processes during the development and progression of cancer [30]. For example, inhibition of MAPK signaling in breast cancer impairs proliferation and promotes apoptosis [31]. Interestingly, this signaling pathway are reported to be regulated by another IncRNA, MALAT1. Han et al. demonstrated that lncRNA MALAT1 can suppress glioma progression through down-regulation of MMP2 and inactivation of MAPK/ERK pathway [32]; Chen et al. revealed that IncRNA MALAT1 promoted
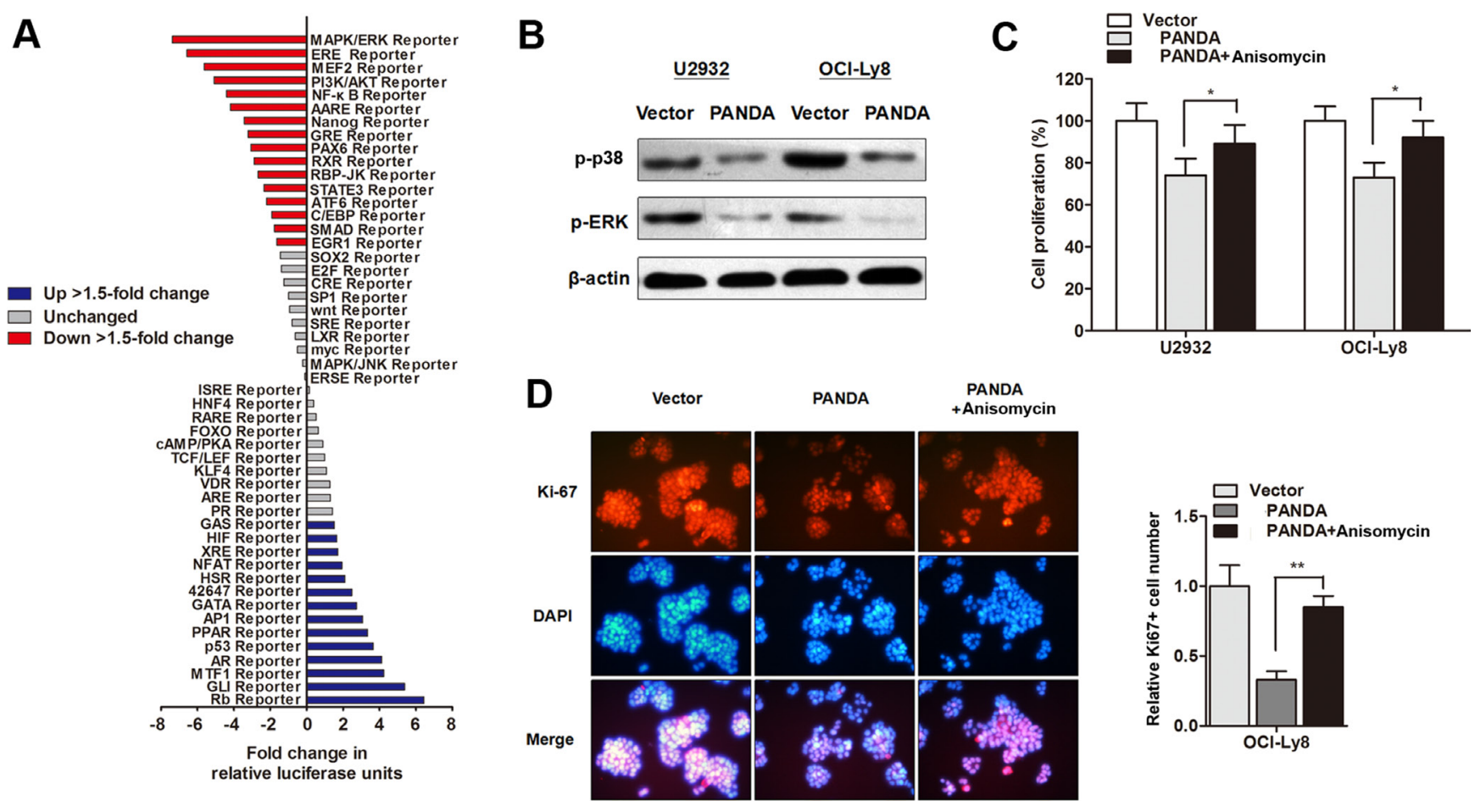

Figure 6: LncRNA PANDA regulates cell proliferation through inactivation of MAPK/ERK signaling pathway. (A) Histogram shows the fold changes for the activities of different signaling pathways, as indicated by reporter activity. (B) Western blot analysis of the expression levels of p38-MAPK and p-ERK protein. (C) CCK8 assay showed that treatment with MAPK/ERK agonist Anisomycin potently abolished PANDA-induced suppression of cell growth. (D) Cell proliferation marker Ki-67 was suppressed by PANDA, however, this effect was partially reversed by MAPK/ERK agonist Anisomycin. 
the activation of MAPK/ERK signaling pathway in N2a cells [33]. These suggest that the MAPK/ERK pathway may also be regulated by other lncRNAs. In our study, we demonstrates that lncRNA PANDA inhibits DLBCL cell growth through the inactivation of MAPK/ERK signaling pathway.

The results of the present study were a little different from others. LncRNA PANDA was reported to be a potential tumor promoter gene in osteosarcoma [15]. But in the present study, it was proved that PANDA acts as a tumor suppressor gene. It may be caused by limited samples or the different types of tumors. Hence, more researches of this lncRNA in other cancers are needed. In conclusion, this is, to our knowledge, the first description of the role of IncRNA PANDA in DLBCL progression. The integrated approach reveals that PANDA is downregulated in DLBCL patients compared with healthy individuals, and closely associated with clinical prognosis. Moreover, it suppresses cell proliferation and caused cellcycle arrest through inactivation of MAPK/ERK signaling pathway. Hence, PANDA may be a promising therapeutic target for patients with DLBCL.

\section{MATERIALS AND METHODS}

\section{Study design}

A multiphase, case-control study was designed to identify lncRNAs as potential biomarkers for differentiating DLBCL and healthy people. Briefly, 114 patients diagnosed with DLBCL but without other diseases and 114 control individuals without the history of DLBCL were recruited from The First Affiliated Hospital of Zhengzhou University. All these participants were allocated to three phases. In the initial screening phase, six serum samples from DLBCL patients and six from healthy controls were subjected to Hiseq sequencing, to identify lncRNAs that significantly differentially expressed. In the training phase, the candidate lncRNAs were firstly verified with RT-qPCR in an independent cohort of 40 primary DLBCL tissues from DLBCL patients and 40 normal lymph node tissues from control individuals with reactive lymph. In the validation phase, the candidate lncRNAs were further validated in another independent group of serum samples obtained from 68 DLBCL patients and 68 controls individuals. The control samples enrolled in this study are matched to the patient samples (tissues and serums) on clinical pathological charicteristics, such as gender and age.

\section{Patients and sample preparation}

All the patients were pathologically confirmed and they were classified according to the tumor-nodemetastasis (TNM) classification. Overall survival (OS) was updated on 1 February 2012 and was defined as the time from inclusion to death for any reason. Recurrence free survival (RFS) was defined as the time from inclusion to recurrence or metastasis progression. After ultrasonic biopsy, tissues specimens were immediately frozen at $-80{ }^{\circ} \mathrm{C}$ until RNA extraction. Venous blood was collected and centrifuged at $4000 \mathrm{rpm}$ for $10 \mathrm{~min}$, within $2 \mathrm{~h}$. The supernatant fluids were then collected and further centrifuged at $12000 \mathrm{rpm}$ for $15 \mathrm{~min}$ to completely remove the cell debris. The whole process was strictly controlled to avoid hemolysis, and the supernatant serum was stored at $-80^{\circ} \mathrm{C}$, until further used.

\section{Hiseq sequencing}

Total tissue RNA was extracted by one-step extraction using a Trizol kit (Life Technologies, USA), and the purity and quantity of RNA were determined by UV spectrophotometry. cDNA library construction and sequencing were performed according to previously described methods (9). Briefly, after extraction of total RNA, ribosomal RNA was separated to isolate as ncRNA as possible. RNA fragments were broken into short fragments randomly. The first chain of cDNA was generated using RNA fragments as templates and 6-bp random primers. Second chain of the cDNA was synthesized according to the kit's instruction (TakaRa Co., Ltd., Dalian, China). After purification, end repair, base $\mathrm{A}$ and sequencing joint adding, the generated cDNA was fragmented using uracil-N-glycosylase (UNG). cDNA fragments were chosen according to size, then PCR amplification was performed to establish the complete sequencing cDNA library. lncRNAs were sequenced using the high-throughput, high-sensitivity HiSeq 2500 sequencing platform (Illumina Company, USA). The whole process and subsequent data analysis were performed by Kangchen Bio-tech, Shanghai, P.R. China. FastQC software was used for quality control of the pretreated data.

\section{Cell culture}

The human DLBCL cell lines (U2932, SUDHL-6, SUDHL-3, OCI-Ly3, and OCI-Ly8) and normal B-cell line (WIL2S) were obtained from Type Culture Collection of the Chinese Academy of Sciences (Shanghai, China). DLBCL cells were cultured in RPMI 1640 (Thermo Fisher Scientific, Wilmington, DE, USA) containing $10 \%$ fetal bovine serum (FBS) (Sigma-Aldrich, St. Louis, MO, USA), $100 \mathrm{U} / \mathrm{ml}$ penicillin, and $100 \mathrm{~g} / \mathrm{ml}$ streptomycin (Life Technologies, Grand Island, NY, USA). B-cell lines were maintained in Iscove's modified medium supplemented with 10\% human serum (NABI Biopharmaceuticals, Boca Raton, FL, USA), 100 U/ml penicillin, $100 \mathrm{mg} / \mathrm{ml}$ streptomycin, and $2 \mathrm{mM}$ l-glutamine (Invitrogen, Carlsbad, CA, USA). All the cells were incubated in a humidified atmosphere at $37^{\circ} \mathrm{C}$ in $5 \% \mathrm{CO}_{2}$ 
and 95\% air. The MAKP/ERK agonist Anisomycin was bought from Sigma-Aldrich (St. Louis, MO, USA) and used as instructions.

\section{RNA oligoribonucleotides and cell transfection}

The small interfering RNAs (siRNA) that specifically target human lncRNA PANDA were designated as si-PANDA (Genechem corporation, Shanghai, China). The lncRNA PANDA overexpression plasmid (PANDA vector), p53 overexpression plasmid or control vector was purchased from Addgene. DLBCL cells were plated in 24-well plates at $1 \times 10^{5}$ per well. Forty-eight hours after plating, $100 \mathrm{nM}$ of RNA oligoribonucleotides were transfected into the cells with Lipofectamine 2000 (Invitrogen) according to the manufacturer's instructions.

\section{RNA extraction, reverse transcription, and RT- qPCR}

Total RNA was isolated from primary DLBCL tissues or cell lines using TRIzol reagent (Invitrogen). And then, the cDNA was synthesized from $200 \mathrm{ng}$ extracted total RNA using the PrimeScript RT reagent Kit (Takara Bio Company, Shiga, Japan) and amplified by RT-qPCR with an SYBR Green Kit (Takara Bio Company) on an ABI PRISM 7500 Sequence Detection System (Applied Biosystems, Foster City, CA, USA) with the housekeeping gene GAPDH as an internal control. The $2^{-\Delta \Delta \mathrm{Ct}}$ method was used to determine the relative quantification of gene expression levels. All the premier sequences were synthesized by RiboBio, and the premier sequences were as follows: PANDA: (Forward) 5'-AGACCCCAGTGGCACCTGAC-3', (Reverse) 5'-GG GCAGAACTTGGCATGATG-3'; p53 (Forward) 5'-GTCG ATCGTCGATCGCTACGC-3', (Reverse) 5'-CGTAG CTAGTCGATCGACTAGC-3'; GAPDH (Forward) 5'-G CACCGTCAAGGCTGAGAAC-3', (Reverse) 5'-ATGGT GGTGAAGACGCCAGT-3'. Each experiment was performed in triplicate.

\section{Signal transduction reporter array}

Cignal Signal Transduction Reporter Array (Qiagen, Valencia, CA, USA) was used to simultaneously investigate alternations in the activities of 50 canonical signalling pathways in response to UCA1 knockdown. Cells were transfected with antisense oligonucleotidestargeting UCA1 for $24 \mathrm{~h}$ and were subsequently transfected with a mixture of a transcription factorresponsive firefly luciferase reporter and a constitutively expressing Renilla construct. The relative activity of each pathway was decided by luciferase/Renilla and normalized by untreated controls. Experiments were performed in triplicates.

\section{Cell proliferation assay}

Cell proliferation was quantified by using the Cell Counting Kit-8 (CCK-8, Beyotime Corporation, Shanghai, China). Briefly, $100 \mu \mathrm{l}$ of cells from the different transfection groups were seeded onto a 96-well plate at a concentration of 2000 cells per well and were incubated at $37^{\circ} \mathrm{C}$. At different time point, the optical density was measured at $450 \mathrm{~nm}$ using a microtiter plate reader, and the rate of cell survival was expressed as the absorbance. The results represent the mean of three replicates under the same conditions.

\section{Cell cycle assay}

Cells were washed in PBS and fixed in $70 \%$ ethanol at $4^{\circ} \mathrm{C}$ for $2 \mathrm{~h}$. DNA staining was done with 10 $\mathrm{mg}$ propidium iodide/mL PBS and $2.5 \mathrm{Ag}$ DNase-free RNase/mL PBS for at least 30 min before flow cytometry in a Coulter EPICS XL flow cytometer (Beckman Coulter, Inc., Fullerton, CA). Cell cycle profiles were generated from flow cytometry analysis with Modifit software (BD Biosciences).

\section{Immunofluorescence analysis}

DLBCL cells were grown to $40 \%$ to $50 \%$ confluence and then transfected with $100 \mathrm{nM}$ of si-PANDA or PANDA overexpression vector. After 48 hours of incubation, the cells were fixed with $4 \%$ paraformaldehyde and permeabilized in $0.2 \%$ Triton X-100 (Sigma-Aldrich) for 20 minutes. The cells were then blocked with $10 \%$ goat serum in PBS for $1 \mathrm{~h}$. Cells were incubated with primary anti-Ki-67 (Cell Signaling Technology) overnight at $4^{\circ} \mathrm{C}$ and then incubated with the appropriate rhodamineconjugated secondary antibody for $1 \mathrm{~h}$. The cells were then washed and incubated with DAPI (Invitrogen) for nuclear staining. The slides were visualized for immunofluorescence with a laser scanning Olympus microscope.

\section{Western blot and antibodies}

The primary antibodies were rabbit anti-human p53 antibody (\#9282T, 1:1000; Cell Signaling Technology, Beverly, MA, USA), p38-MAPK antibody (\#8690T, 1:1000; Cell Signaling Technology), rabbit anti-human phospho-ERK1/2 antibody (\#4695T, 1:1000; Cell Signaling Technology), and rabbit anti-human $\beta$-actin antibody (\#4970T, 1:1000; Cell Signaling Technology). Horseradish peroxidase-conjugated (HRP) anti-rabbit antibodies (1:5000; Santa Cruz Biotechnology, Santa Cruz, CA, USA) were used as the secondary antibodies. Cell lysates in $1 \times$ SDS loading buffer $(60 \mathrm{mM}$ Tris $-\mathrm{HCl}, \mathrm{pH}$ $6.8 ; 2 \%$ SDS; $20 \%$ glycerol; $0.25 \%$ bromophenol blue; and $1.25 \%$ 2-mercaptoethanol) were incubated at $100{ }^{\circ} \mathrm{C}$ 
for $10 \mathrm{~min}$ to facilitate sample loading for conventional western blotting analysis. The relative protein levels were quantified using densitometry with a Gel-Pro Analyzer (Media Cybernetics, Rockville, MD, USA).

\section{Chromatin immunoprecipitation (ChIP)}

ChIP was performed using the EZ ChIP ${ }^{\mathrm{TM}}$ Chromatin Immunoprecipitation Kit (Millipore, Bedford, MA, USA), according to the manufacturer's protocol. Briefly, cross-linked chromatin was sonicated into 200 $1000 \mathrm{bp}$ fragments. The chromatin located on the promoter of IncRNA PANDA was immunoprecipitated by using anti-p53 (Cell Signaling Technology) antibodies. An isotype-matched IgG was used as a negative control, and the total RNA that immunoprecipitated by p53 antibody served as a positive control. RT-qPCR was conducted to detect the relative enrichment of the lncRNA PANDA promoter. The ChIP primer sequences are as follows: (forward) 5'-GCCCACATAAACACCTCACAAATGA3' and (reverse) 5'-CCT TGGAAGCCGGGAGTAGCT-3' were used for amplify the promoter region of PANDA.

\section{Statistical analysis}

The differences of IncRNA or mRNA expression level between different groups were analyzed by the Mann-Whitney U-test or Kruskal-Wallis test. A log-rank test was used to analyze the statistical differences in survival as deduced from Kaplan-Meier curves. Count dates were described as frequency and examined using Fisher's exact test. All differences were regarded as statistically significant when $P<0.05$. Statistical analyses were performed with GraphPad Prism 5.01 (GraphPad Software, La Jolla, CA, USA).

\section{CONFLICTS OF INTEREST}

We declare no potential conflicts of interest.

\section{REFERENCES}

1. Tilly H, Gomes da Silva M, Vitolo U, Jack A, Meignan M, Lopez-Guillermo A, Walewski J, Andre M, Johnson PW, Pfreundschuh M, Ladetto M. Diffuse large B-cell lymphoma (DLBCL): ESMO Clinical Practice Guidelines for diagnosis, treatment and follow-up. Ann Oncol. 2015; 26:v116-125.

2. Smith A, Howell D, Patmore R, Jack A, Roman E. Incidence of haematological malignancy by sub-type: a report from the Haematological Malignancy Research Network. Br J Cancer. 2011; 105:1684-1692.

3. Feugier $\mathrm{P}$, Van Hoof A, Sebban C, Solal-Celigny $\mathrm{P}$, Bouabdallah R, Ferme C, Christian B, Lepage E, Tilly H, Morschhauser F, Gaulard P, Salles G, Bosly A, et al. Longterm results of the R-CHOP study in the treatment of elderly patients with diffuse large B-cell lymphoma: a study by the Groupe d'Etude des Lymphomes de l'Adulte. J Clin Oncol. 2005; 23:4117-4126.

4. Lee HJ, Shin DH, Kim KB, Shin N, Park WY, Lee JH, Choi KU, Kim JY, Lee CH, Sol MY. Polycomb protein EZH2 expression in diffuse large B-cell lymphoma is associated with better prognosis in patients treated with rituximab, cyclophosphamide, doxorubicin, vincristine and prednisone. Leuk Lymphoma. 2014; 55:2056-2063.

5. Vaidya R, Witzig TE. Prognostic factors for diffuse large B-cell lymphoma in the R(X)CHOP era. Ann Oncol. 2014; 25:2124-2133.

6. Djebali S, Davis CA, Merkel A, Dobin A, Lassmann T, Mortazavi A, Tanzer A, Lagarde J, Lin W, Schlesinger F, Xue C, Marinov GK, Khatun J, et al. Landscape of transcription in human cells. Nature. 2012; 489:101-108.

7. Tsai MC, Spitale RC, Chang HY. Long intergenic noncoding RNAs: new links in cancer progression. Cancer Res. 2011; 71:3-7.

8. Gibb EA, Brown CJ, Lam WL. The functional role of long non-coding RNA in human carcinomas. Mol Cancer. 2011; $10: 38$.

9. Iyer MK, Niknafs YS, Malik R, Singhal U, Sahu A, Hosono Y, Barrette TR, Prensner JR, Evans JR, Zhao S, Poliakov A, Cao X, Dhanasekaran SM, et al. The landscape of long noncoding RNAs in the human transcriptome. Nat Genet. 2015; 47:199-208.

10. White NM, Cabanski CR, Silva-Fisher JM, Dang HX, Govindan R, Maher CA. Transcriptome sequencing reveals altered long intergenic non-coding RNAs in lung cancer. Genome Biol. 2014; 15:429.

11. Xue X, Yang YA, Zhang A, Fong KW, Kim J, Song B, Li S, Zhao JC, Yu J. LncRNA HOTAIR enhances ER signaling and confers tamoxifen resistance in breast cancer. Oncogene. 2016; 35:2746-2755.

12. Gutschner T, Hammerle M, Eissmann M, Hsu J, Kim Y, Hung G, Revenko A, Arun G, Stentrup M, Gross M, Zornig M, MacLeod AR, Spector DL, et al. The noncoding RNA MALAT1 is a critical regulator of the metastasis phenotype of lung cancer cells. Cancer Res. 2013; 73:1180-1189.

13. Peng W, Gao W, Feng J. Long noncoding RNA HULC is a novel biomarker of poor prognosis in patients with pancreatic cancer. Med Oncol. 2014; 31:346.

14. Mattick JS. RNA regulation: a new genetics? Nat Rev Genet. 2004; 5:316-323.

15. Kotake Y, Goto T, Naemura M, Inoue Y, Okamoto H, Tahara K. Long Noncoding RNA PANDA Positively Regulates Proliferation of Osteosarcoma Cells. Anticancer Res. 2017; 37:81-85.

16. Yan Y, Han J, Li Z, Yang H, Sui Y, Wang M. Elevated RNA expression of long noncoding HOTAIR promotes cell proliferation and predicts a poor prognosis in patients with diffuse large B cell lymphoma. Mol Med Rep. 2016; 13:5125-5131. 
17. Peng $\mathrm{W}, \mathrm{Wu}$ J, Feng J. Long noncoding RNA HULC predicts poor clinical outcome and represents pro-oncogenic activity in diffuse large B-cell lymphoma. Biomed Pharmacother. 2016; 79:188-193.

18. Peng W, Feng J. Long noncoding RNA LUNAR1 associates with cell proliferation and predicts a poor prognosis in diffuse large B-cell lymphoma. Biomed Pharmacother. 2016; 77:65-71.

19. Peng W, Wu J, Feng J. LincRNA-p21 predicts favorable clinical outcome and impairs tumorigenesis in diffuse large B cell lymphoma patients treated with R-CHOP chemotherapy. Clin Exp Med. 2017; 17:1-8.

20. Peng W, Fan H, Wu G, Wu J, Feng J. Upregulation of long noncoding RNA PEG10 associates with poor prognosis in diffuse large $\mathrm{B}$ cell lymphoma with facilitating tumorigenicity. Clin Exp Med. 2016; 16:177-182.

21. $\mathrm{Wu}$ GS. The functional interactions between the p53 and MAPK signaling pathways. Cancer Biol Ther. 2004; $3: 156-161$.

22. Hung T, Wang Y, Lin MF, Koegel AK, Kotake Y, Grant GD, Horlings HM, Shah N, Umbricht C, Wang P, Wang Y, Kong B, Langerod A, et al. Extensive and coordinated transcription of noncoding RNAs within cell-cycle promoters. Nat Genet. 2011; 43:621-629.

23. de Kok JB, Verhaegh GW, Roelofs RW, Hessels D, Kiemeney LA, Aalders TW, Swinkels DW, Schalken JA. DD3(PCA3), a very sensitive and specific marker to detect prostate tumors. Cancer Res. 2002; 62:2695-2698.

24. Zhou M, Zhao H, Xu W, Bao S, Cheng L, Sun J. Discovery and validation of immune-associated long non-coding RNA biomarkers associated with clinically molecular subtype and prognosis in diffuse large B cell lymphoma. Mol Cancer. 2017; 16:16.

25. Levine AJ, Hu W, Feng Z. The P53 pathway: what questions remain to be explored? Cell Death Differ. 2006; 13:1027-1036.

26. Puvvula PK, Desetty RD, Pineau P, Marchio A, Moon A, Dejean A, Bischof O. Long noncoding RNA PANDA and scaffold-attachment-factor SAFA control senescence entry and exit. Nat Commun. 2014; 5:5323.
27. Zhu JJ, Luo J, Wang W, Yu K, Wang HB, Shi HB, Sun YT, Lin XZ, Li J. Inhibition of FASN reduces the synthesis of medium-chain fatty acids in goat mammary gland. Animal. 2014; 8:1469-1478.

28. Morachis JM, Murawsky CM, Emerson BM. Regulation of the p53 transcriptional response by structurally diverse core promoters. Genes Dev. 2010; 24:135-147.

29. Mahadevan D, Islam S, Qi W, Morales C, Cooke L, Spier C, Weterings E. Disruption of Aneuploidy and Senescence induced by Aurora inhibition promotes intrinsic Apoptosis in double hit or double expressor Diffuse Large B-cell Lymphomas. Mol Cancer Ther. 2017.

30. Vega GG, Aviles-Salas A, Chalapud JR, MartinezPaniagua M, Pelayo R, Mayani H, Hernandez-Pando R, Martinez-Maza O, Huerta-Yepez S, Bonavida B, Vega MI. P38 MAPK expression and activation predicts failure of response to CHOP in patients with Diffuse Large B-Cell Lymphoma. BMC Cancer. 2015; 15:722.

31. Jin YC, Li ZH, Hong ZS, Xu CX, Han JA, Choi SH, Yin JL, Zhang QK, Lee KB, Kang SK, Song MK, Kim YJ, Kang HS, et al. Conjugated linoleic acid synthesis-related protein proteasome subunit alpha 5 (PSMA5) is increased by vaccenic acid treatment in goat mammary tissue. J Dairy Sci. 2012; 95:4286-4297.

32. Han $Y$, Wu Z, Wu T, Huang Y, Cheng Z, Li X, Sun T, Xie X, Zhou Y, Du Z. Tumor-suppressive function of long noncoding RNA MALAT1 in glioma cells by downregulation of MMP2 and inactivation of ERK/MAPK signaling. Cell Death Dis. 2016; 7:e2123.

33. Chen L, Feng P, Zhu X, He S, Duan J, Zhou D. Long noncoding RNA Malat1 promotes neurite outgrowth through activation of ERK/MAPK signalling pathway in N2a cells. J Cell Mol Med. 2016; 20:2102-2110. 